\title{
PRODUCCIÓN Y CALIDAD FISICOQUÍMICA DE LECHE DE CABRAS SUPLEMENTADAS CON FORRAJE VERDE HIDROPÓNICO DE MAÍZ ${ }^{1}$
}

\author{
Mario García-Carrillo², Lilia Salas-Pérez ${ }^{3}$, Juan Ramón Esparza-Rivera ${ }^{4}$, Pablo Preciado-Rangel ${ }^{5}$, \\ Jaime Romero-Paredes ${ }^{2}$
}

\begin{abstract}
RESUMEN
Producción y calidad fisicoquímica de leche de cabras suplementadas con forraje verde hidropónico de maíz. El objetivo del presente trabajo fue evaluar la producción y calidad fisicoquímica de leche de cabras al ser suplementadas con dietas que contenían forraje verde hidropónico de maíz. Este estudio se desarrolló en la Comarca Lagunera de Coahuila, México durante los meses de marzo a agosto de 2011. Para la dieta 1 se empleó una relación 85:15 (base seca) de alfalfa henificada y forraje verde hidropónico (AH: FVH). La dieta 2 se formuló con una relación 70:30 de AH y FVH, mientras que la dieta testigo tuvo una relación 87.5:12.5 de alfalfa henificada y vaina de mezquite. Se utilizó un diseño en triple cuadro latino. La producción y calidad de leche fueron mayores al suministrar la dieta compuesta con alfalfa henificada y forraje verde hidropónico de maíz al 30\%.
\end{abstract}

Palabras clave: forraje verde hidropónico, leche de cabra, dietas formuladas.

\begin{abstract}
Yielding and physicochemical quality of milk of goat supplemented with hydroponic green corn fodder. The purpose of this investigation was to evaluate the yield and physicochemical quality of goat milk obtained under supplementation of diets containing hydroponic green fodder. This study was performed in the Comarca Lagunera in Coahuila, México, from March to August of 2011. For diet 1 a ratio of 85:15 (dry matter) of alfalfa and hydroponic green fodder (AH:FVH) was employed. Diet 2 was formulated at a 70:30 rate of alfalfa and $\mathrm{FVH}$; meanwhile the control diet contained 87.5:12.5 of alfalfa and mezquite sheath. A triple latin square design was used for the experiment. The highest milk yield and quality was obtained from supplementing the goats diet with the alfalfa and $30 \%$ of hydroponic green fodder.
\end{abstract}

Key words: hydroponic green fodder, milk goat, formulated diets.

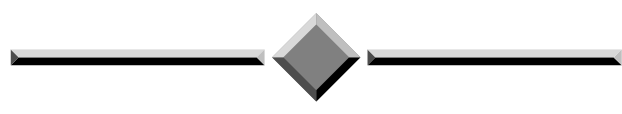

1 Recibido: 2 de febrero, 2012. Aceptado: 1 de abril, 2013. Parte del proyecto "Forraje Hidropónico Orgánico de maíz y su respuesta en la producción y calidad de leche de cabra", Universidad Autónoma Agraria Antonio Narro-Unidad Laguna (UAAAN-UL).

2 Universidad Autónoma Agraria Antonio Narro Unidad Laguna. Periférico Raúl López Sánchez Km. 2. 27059, Torreón, Coahuila, México. mgc570118@hotmail.com,romeropj@yahoo.com

3 Universidad Politécnica de Gómez Palacio. Carretera el Vergel-La Torreña km. 0820, Gómez Palacio, Durango, México. lilia-nt@hotmail. com, lisape95@hotmail.com (Autor para correspondencia).

4 Facultad de Ciencias Químicas, Unidad Gómez Palacio, Universidad Juárez del Estado de Durango, Gómez Palacio, Durango, México. jresparza02001@yahoo.com

5 Instituto Tecnológico de Torreón, Carretera Torreón-San Pedro Km. 7.5, Torreón Coahuila, México. ppreciador@yahoo.com.mx 


\section{INTRODUCCIÓN}

En la parte norte de México se ha tenido desabasto de forraje convencional en la última década como consecuencia de fenómenos climatológicos tales como sequías y heladas, lo cual ha afectado negativamente la producción agropecuaria (Vargas 2008, Rivera et al. 2010). Por otra parte, la Comarca Lagunera, región ubicada entre los estados de Durango y Coahuila en el norte del país, es la mayor cuenca lechera a nivel nacional además de ser una importante zona productora de carne bovina y de ave (SIAP 2010). Asimismo, en esta región se ha promovido la explotación de ganado caprino tanto para producción de carne como de leche, resaltando que la leche de cabra es un producto nutritivo y económico dirigido principalmente a personas convalecientes y/o alérgicas a otras leches (Sánchez et al. 2003). Sin embargo, se ha reportado que $70 \%$ del inventario caprino de la Comarca Lagunera, el cual consta de 600 mil cabezas (SIAP 2011), se encuentra en áreas marginadas de temporal donde el pastoreo es el principal sistema de producción (García et al. 2005), teniéndose desabasto de forraje para alimentación de este ganado en algunas épocas del año. Así pues, es requerido el planteamiento de alternativas de producción de forrajes, que permitan la disponibilidad de alimento para animales durante todo el año, a la vez que minimice el uso del agua y de agroquímicos en su producción. El cultivo de forraje en medio hidropónico, puede ser una alternativa para la alimentación animal, permitiendo cultivar especies altamente productivas en medios artificiales (Rodríguez 2003).

El forraje verde hidropónico (FVH) consiste en la germinación de semillas de gramíneas o leguminosas, seguido del crecimiento de las plantas bajo condiciones ambientales controladas de luz, temperatura y humedad en ausencia del suelo (FAO 2001). El FVH puede ser utilizado para la alimentación de rumiantes, cerdos, conejos y aves (Herrera et al. 2007). Además, este forraje ofrece una serie de ventajas, que incluyen el desarrollo del cultivo en áreas pequeñas y producción continua durante todo el año, así como buena calidad bromatológica sin que se tengan reportes de trastornos digestivos en ganado atribuibles a su consumo (González et al. 2006, Salas-Pérez et al. 2012). Una de las plantas más utilizadas con fines forrajeros es el maíz, debido principalmente a su elevado valor nutritivo y rendimiento (Elizondo y Boschini 2002), lo cual permite que en sistemas hidropónicos se obtengan altos volúmenes de FVH de maíz.

El objetivo del presente estudio fue evaluar la producción y calidad fisicoquímica de leche de cabras al ser suplementadas con dietas que contenían forraje verde hidropónico de maíz. El testigo incluyó vaina de mezquite.

\section{MATERIALES Y MÉTODOS}

\section{Localización}

El trabajo se realizó en la granja caprina "Espialon", ubicada en la Comarca Lagunera en Francisco I. Madero, Coahuila (México), durante los meses de marzo a agosto de 2011, con coordenadas geográficas: $24^{\circ} 30^{\prime}$ y $27^{\circ} \mathrm{N}$, y $102^{\circ} 00^{\prime}$ y $104^{\circ} 40^{\prime} \mathrm{O}$, altitud de 1100 msnm; precipitación media anual de $240 \mathrm{~mm}$ y clima árido seco (Villanueva et al. 2011).

\section{Producción del forraje verde hidropónico}

El forraje verde hidropónico (FVH) de maíz se cultivó en un invernadero tipo túnel automatizado cubierto de plástico con un área de $144 \mathrm{~m}^{2}$. La temperatura interna del invernadero fue de $29^{\circ} \mathrm{C}$ con humedad relativa de $75 \%$. Se utilizaron semillas de maíz (Zea mays L.) variedad criolla "San Lorenzo", con una germinación de $90 \%$, y libre de agroquímicos. Estas fueron limpiadas inicialmente eliminando las que presentaban daños o estaban quebradas, y luego fueron desinfectadas con hipoclorito de sodio $(1 \mathrm{ml} / \mathrm{l})$, enjuagándolas finalmente con agua potable (Rodríguez 2003).

La semilla desinfectada fue sometida posteriormente a una etapa de pre germinación mediante inmersión en una solución de $\mathrm{Ca}(\mathrm{OH})_{2}$ en agua potable $(1 \mathrm{~g} / \mathrm{l})$ a temperatura ambiente $\left(25 \mathrm{a} 28^{\circ} \mathrm{C}\right)$ durante $24 \mathrm{~h}$ para escarificar la semilla y eliminar patógenos (López et al. 2009). Después, las semillas fueron enjuagadas con agua potable para eliminar el exceso de $\mathrm{Ca}(\mathrm{OH})_{2}$, y escurridas antes de la etapa de germinación, la cual consistió en la colocación de las pre germinadas en botes de plástico de $201 \mathrm{con}$ perforaciones para permitir suficiente aireación. Los botes con semilla en germinación fueron cubiertos con plástico negro y colocados en un cuarto oscuro, seleccionando semillas germinadas con radículas de 1 a 1,5 $\mathrm{cm}$ de longitud después de $24 \mathrm{~h}$ de germinación. 
Las semillas germinadas fueron colocadas en bandejas de $35 \times 53 \times 5 \mathrm{~cm}$, con una densidad de $3,5 \mathrm{~kg} / \mathrm{m}^{2}$ (Salas-Pérez et al. 2010). Se tomó al día de siembra como el inicio del tiempo de crecimiento de las plantas (día 0). El riego de las plántulas se realizó mediante aspersión con agua sin aplicación de fertilizantes, con una dosis de 9,87 1/ $\mathrm{m}^{2} /$ día durante el desarrollo del cultivo desde el día 0 hasta el día 14 de cosecha.

\section{Selección de los animales}

Se seleccionaron para el experimento nueve cabras de raza Saanen multíparas de tres años de edad y tres partos sencillos, con $180 \pm 20$ días de etapa productiva de leche. Previamente al estudio los animales fueron desparasitados con ivermectina $(2 \mathrm{mg})$ suministrando una tableta por cada $10 \mathrm{~kg}$ de peso en ayunas; asimismo fueron vitaminados con una solución oleosa para uso parenteral compuesta de vitaminas A, $\mathrm{D}_{3}$ y vitamina $\mathrm{E}$, administrando $1 \mathrm{ml}$ por animal. Se tomó el peso de cada uno de ellos seleccionándose cabras de $40 \pm 2 \mathrm{~kg}$ con producción lechera de 1,5 a 1,8 litros diarios. Posteriormente los animales fueron identificados y distribuidos aleatoriamente en corrales individuales techados. Por otra parte, durante las dos semanas previas al inicio del trabajo experimental los animales tuvieron acceso a una dieta a base de alfalfa henificada con gradual incorporación de forraje verde hidropónico para facilitar la adaptación a las dietas tratamiento. Asimismo las cabras tuvieron acceso de agua ad libitum.

\section{Formulación de las dietas}

Se formularon dos dietas conteniendo forraje verde hidropónico a diferente nivel, y un tratamiento o dieta testigo que incluyó vaina de mezquite (Prosopis glandulosa). Las tres contenían alfalfa henificada y fueron proporcionadas a las cabras ajustando las cantidades ofrecidas de manera individual en base materia seca, tomando como referencia el $5 \%$ del peso vivo (De la Rosa 2011, Revidatti et al. 2012) (Cuadro 1).

Se consideró que las dietas formuladas cubrieran los requerimientos diarios proteicos recomendados para cabras lecheras (NCR 2007, Elizondo-Salazar 2008). En lo referente a los contenidos de fibra ácido detergente y fibra neutro detergente (FAD y FND), los valores de los ingredientes usados en el estudio estuvieron dentro de lo recomendado por Aregherore
Cuadro 1. Formulación de dietas suplementadas* a cabras en lactancia. Francisco I. Madero, Coahuila, México. 2011.

\begin{tabular}{lccc}
\hline & \multicolumn{3}{c}{ g de materia seca } \\
\cline { 2 - 4 } & AH & FVH & VM \\
\hline Dieta 1 (AH-FVH 15\%) & 1700 & 300 & \\
Dieta 2 (AH-FVH 30\%) & 1400 & 600 & \\
Dieta testigo (AH-VM) & 1745 & & 255 \\
\hline
\end{tabular}

$\mathrm{AH}=$ alfalfa henificada, $\mathrm{FVH}=$ forraje verde hidropónico, $\mathrm{VM}=$ vaina de mezquite.

* Raciones de dietas formuladas con base en los requerimientos diarios de $2000 \mathrm{~g}$ de materia seca por animal representando el 5\% en peso vivo (De la Rosa 2011, Revidatti et al. 2012).

et al. (2006) para FAD, y Herrera et al. (2007) para FND (Cuadro 2).

Para la dieta 1 se empleó una relación 85:15 (base seca) de alfalfa henificada y forraje verde hidropónico (AH:FVH). La dieta 2 se formuló con una relación 70:30 de $\mathrm{AH}$ y $\mathrm{FVH}$, mientras que la dieta testigo tuvo una relación 87.5:12.5 de alfalfa henificada y vaina de mezquite (AH:VM). Las relaciones 85:15 y 70:30 se formularon de acuerdo a lo recomendado por FAO (2001) que indica un consumo diario de $300 \mathrm{~g}$ de materia seca proveniente de forraje verde hidropónico para mejorar la productividad en cabras. Por otro lado, la vaina de mezquite fue incluida en la dieta testigo debido a que la mayoría de las explotaciones caprinas

Cuadro 2. Composición química de los ingredientes de las dietas suplementadas en cabras en lactancia. Francisco I. Madero, Coahuila, México. 2011.

\begin{tabular}{lccc}
\hline Parámetro & AH & FVH & VM \\
\hline Humedad & 10,0 & 85,0 & 15,0 \\
Materia seca & 90,0 & 15,0 & 85,0 \\
\hline Parámetro (\% base seca) & & & \\
\hline Proteína cruda & 8,0 & 15,5 & 9,6 \\
Fibra ácido detergente & 42,1 & 14,4 & 39,6 \\
Fibra neutro detergente & 54,3 & 38,4 & 48,1 \\
Grasa & 2,0 & 6,9 & 3,5 \\
Ceniza & 2,9 & 4,3 & 4,5 \\
\hline
\end{tabular}

$\mathrm{FVH}=$ forraje verde hidropónico, $\mathrm{AH}=$ alfalfa henificada, $\mathrm{VM}=$ vaina de mezquite. 
de esta región de México no incluyen concentrados y sales en las dietas para cabras debido a los altos costos, pero tienen posibilidad de obtener vaina de mezquite durante algunas épocas del año. La composición bromatológica de los ingredientes de las dietas (Cuadro 2) se obtuvo mediante los métodos oficiales de la AOAC (2000) para materia seca, proteína cruda, cenizas y grasa. Las fibras ácido y neutro detergente se cuantificaron con el método de fraccionamiento con detergente y filtración subsecuente (Van Soest et al. 1991). Asimismo, se determinó la composición química de las dietas suplementadas (Cuadro 3).

Cuadro 3. Composición bromatológica de dietas suplementadas a cabras en lactancia. Francisco I. Madero, Coahuila, México. 2011.

\begin{tabular}{lccc}
\hline & \multicolumn{3}{c}{ Porcentaje base seca } \\
\cline { 2 - 4 } & Dieta 1 & Dieta 2 & Dieta testigo \\
Componente & $\mathbf{1 5 \%}$ & $\mathbf{3 0 \%}$ & \\
\hline Proteína & 11,9 & 13,4 & 8,2 \\
FAD & 33,8 & 30,6 & 38,8 \\
FND & 40,2 & 39,4 & 48,5 \\
Grasa & 4,5 & 5,5 & 2,2 \\
Ceniza & 3,6 & 3,9 & 3,1 \\
\hline
\end{tabular}

$\mathrm{FVH}=$ forraje verde hidropónico, $\mathrm{AH}=$ alfalfa henificada, $\mathrm{VM}=$ vaina de mezquite, $\mathrm{FAD}=$ fibra ácido resistente, $\mathrm{FND}=$ fibra neutro detergente.

\section{Consumo de alimento}

Las raciones de alimento fueron ofrecidas dos veces al día: una mitad por la mañana (8:00), y la otra por la tarde (17:00), teniendo los animales agua a su disposición permanentemente. La adaptación previa al consumo de alimento permitió modificar el consumo voluntario observándose que los animales fueron consistentes y no mostraron evidencias de insatisfacción. El consumo de alimento en g de materia seca se obtuvo mediante la diferencia entre el alimento ofrecido y el sobrante no consumido (Cuadro 4).

\section{Variables evaluadas}

A cada animal evaluado se le asignó un tratamiento durante un periodo de catorce días, con once iniciales de adaptación y los siguientes tres días de evaluación. Se realizaron tres periodos diferentes y en cada uno se aplicó la suplementación de la dieta correspondiente. Las variables medidas fueron: producción y calidad de leche (acidez, grasa, proteína, caseína, peso específico, sólidos no grasos y sólidos totales).

Producción de leche. La producción de leche se midió en litros, realizándose una ordeña manual a las 6:00 am, los días de inicio de cada periodo y durante los días de evaluación (tres días), en cada uno de los periodos para determinar si había variaciones por la suplementación.

Calidad de leche. Las pruebas analíticas para determinar la calidad fisicoquímica de la leche, fueron realizadas antes y después de la suplementación de dietas con el objetivo de evaluar cambios en la composición nutricional de la leche, atribuibles a la suplementación de las dietas formuladas. Para los análisis químicos fueron tomadas muestras de 500 $\mathrm{ml}$ de leche en recipientes plásticos estériles con tapa, las cuales fueron trasladadas inmediatamente en contenedores térmicos al laboratorio. Los análisis químicos se realizaron siguiendo métodos oficiales de la AOAC (2000). Para determinación de acidez se empleó el método 947.05 consistente en la titulación de una alícuota de $9 \mathrm{ml}$ de muestra utilizando como

Cuadro 4. Consumo de las dietas suplementadas en cabras lecheras. Francisco I. Madero, Coahuila, México. 2011.

\begin{tabular}{lccc}
\hline & $\begin{array}{c}\text { Dieta 1 } \\
\text { AH-FVH 15\% }\end{array}$ & $\begin{array}{c}\text { Dieta 2 } \\
\text { AH-FVH 30\% }\end{array}$ & $\begin{array}{c}\text { Dieta testigo } \\
\text { AH-VM }\end{array}$ \\
\hline Consumo de materia seca (kg/animal/día) & $1,770 \pm 0,088$ & $1,960 \pm 0,098$ & $1,700 \pm 0,085$ \\
\hline
\end{tabular}

$\mathrm{FVH}=$ forraje verde hidropónico, $\mathrm{AH}=$ alfalfa henificada, $\mathrm{VM}=$ vaina de mezquite. 
valorante $\mathrm{NaOH} 0,1 \mathrm{~N}$. El contenido de grasa se determinó mediante el método turbidimétrico (969.16). La proteína se determinó por el método 920.123 mediante determinación de nitrógeno para después calcularlo como porcentaje de proteína. La caseína se analizó mediante el método 927.03, y para sólidos totales se empleo el método 925.23 determinando la gravedad específica de la leche con un lactodensímetro Funke Gerber. Finalmente los sólidos no grasos en leche se calcularon por diferencia entre sólidos totales y el contenido de grasa (990.21), y el peso específico se obtuvo empleando un lactodensímetro Funke Gerber graduado (Chacón 2004).

\section{Diseño experimental y análisis estadístico}

Se utilizó un diseño de cuadrado latino triple, donde se asignaron tres animales por cuadrado en tres repeticiones (Steel y Torrie 1980). Para cada grupo de cabras (repeticiones) se asignó un tratamiento a cada animal durante un periodo de catorce días, con once días iniciales de adaptación y los siguientes tres de evaluación. Se realizaron tres periodos experimentales y al término de cada uno se procedió a cambiar el tratamiento de cada animal dentro de cada cuadrado latino. Se realizó un análisis de varianza mediante el Proc GLM de SAS (SAS 1999) y la prueba de separación de medias se hizo mediante la prueba de Tukey $(\mathrm{p}<0,05)$.

\section{RESULTADOS Y DISCUSIÓN}

\section{Producción de leche}

La producción de leche mostró diferencias significativas entre las dietas $(\mathrm{P}<0,05$, Cuadro 5), donde las cabras suplementadas con la dieta 2 (AH-FVH 30\%) produjeron mayor cantidad de leche que cuando consumieron las dietas 1 y testigo. Estos resultados confirman la importancia de la aceptación de esta sobre el consumo de materia seca y rendimiento de las cabras lecheras (Nunes et al. 2007).

La aceptación del FVH concuerda por lo obtenido por Valdivia (1996), quien realizó pruebas en rumiantes sin problemas de aceptación. Además, el animal al verse en la posibilidad de seleccionar o elegir el alimento ofrecido opta generalmente por aquel verde o fresco, por lo tanto, la cantidad de materia seca consumida es mayor que cuando están consumiendo heno únicamente (Mazorra et al. 2009). Para el caso de la dieta 2 (AH-FVH 30\%) los animales tuvieron mayor proporción de material verde, lo cual pudiera explicar el mayor consumo de este tratamiento. Por otro lado, López et al. (2009) señala que existe una relación directa entre los niveles de materia seca del FVH de maíz y el funcionamiento digestivo de los rumiantes, atribuyendo esto al menor contenido de fibras en este tipo de forraje. Lo anterior conlleva a lo mencionado por Rodríguez (2003), que señala que la calidad del FVH radica en su alto contenido de nutrientes tales como proteínas, minerales, vitaminas y componentes enzimáticos en semillas recién germinadas, tallos y hojas jóvenes. Esto pudo haber afectado la aceptación del forraje por los animales y por ende la mayor producción.

Se han observado aumentos de producción de leche hasta niveles de $20 \%$ en pruebas de producción con ganado lechero (Romero-Valdez et al. 2009). En otra prueba de producción, Lomeli (2000) al proporcionar FVH logró incrementos en la producción de leche en un 4,2\%. La literatura referente a ganado caprino es muy limitada, sin embargo los resultados del presente trabajo concuerdan con las investigaciones mencionadas anteriormente, ya que se obtuvo un

Cuadro 5. Producción de leche en cabras suplementadas con tres diferentes dietas. Francisco I. Madero, Coahuila, México. 2011.

\begin{tabular}{lccc}
\hline & $\begin{array}{c}\text { Dieta 1 } \\
\text { AH-FVH 15\% }\end{array}$ & $\begin{array}{c}\text { Dieta 2 } \\
\text { AH-FVH 30\% }\end{array}$ & $\begin{array}{c}\text { Dieta testigo } \\
\text { AH-VM }\end{array}$ \\
\hline Producción de leche (1/animal/día) & $1,68 \mathrm{~b}$ & $2,06 \mathrm{a}$ & $1,73 \mathrm{~b}$ \\
\hline
\end{tabular}

$\mathrm{FVH}=$ forraje verde hidropónico, $\mathrm{AH}=$ alfalfa henificada, $\mathrm{VM}=$ vaina de mezquite.

Valores seguidos de diferente letra en el mismo renglón indican diferencia significativa según la prueba de Tukey $(\mathrm{P}<0,05)$. 
incremento de leche del $16 \%$ referenciando a la dieta testigo.

\section{Calidad fisicoquímica de leche}

La calidad de la leche depende en gran medida de su composición bromatológica, reportándose que los principales componentes de este producto (proteína, grasa y sólidos totales) dependen de factores que incluyen raza, sistema de alimentación, periodo de lactancia y formas de manejo del rebaño (Pinto et al. 1998). En el presente experimento se obtuvo leche de calidad aceptable en las dietas suplementadas (Ludeña et al. 2006), con acidez y peso específico similar (Cuadro 6). Por otra parte, la leche producida al suministrar la dieta 2 tuvo mayor contenido de caseína, sólidos totales, sólidos no grasos, y de proteína $(\mathrm{P}<0,05)$, que es uno de los indicadores más importantes de la calidad de este producto alimenticio.

El porcentaje en grasa de la leche depende principalmente de la genética del animal y de la naturaleza y composición de la dieta que este recibe, ya que esta determina cambios en la fermentación ruminal, modificando la producción de los distintos ácidos grasos, y con ello el contenido en grasa de la leche (Boza y Sanz-Sampelayo 1997). El contenido de grasa obtenido en el presente experimento se vio afectado significativamente entre tratamientos, siendo mayor la dieta 1 , seguida por la dieta 2 y la dieta testigo (Cuadro 7). Esto pudiera ser explicado de acuerdo a lo señalado por Boza y Sanz-Sampelayo (1997) que mencionan que la modificación de la composición de la leche en los rumiantes es más difícil, que la de los animales monogástricos, debido al proceso de hidrogenación que en el rumen sufren la grasa de los
Cuadro 6. Composición química de la leche de cabras suplementadas con tres diferentes dietas. Francisco I. Madero, Coahuila, México. 2011.

\begin{tabular}{lccc}
\hline Parámetro & $\begin{array}{c}\text { AH-FVH } \\
\mathbf{1 5 \%}\end{array}$ & $\begin{array}{c}\text { AH-FVH } \\
\mathbf{3 0 \%}\end{array}$ & AH-VM \\
\hline Acidez (\%) & $0,18 \mathrm{a}$ & $0,18 \mathrm{a}$ & $0,18 \mathrm{a}$ \\
Grasa (\%) & $4,23 \mathrm{a}$ & $3,92 \mathrm{~b}$ & $3,65 \mathrm{~b}$ \\
Proteína (\%) & $3,54 \mathrm{~b}$ & $3,68 \mathrm{a}$ & $3,58 \mathrm{~b}$ \\
Caseína (\%) & $2,99 \mathrm{~b}$ & $3,06 \mathrm{a}$ & $2,95 \mathrm{~b}$ \\
Sólidos totales & $11,65 \mathrm{~b}$ & $12,35 \mathrm{a}$ & $11,98 \mathrm{~b}$ \\
$(\%)$ & & & \\
Sólidos no grasos & $8,06 \mathrm{~b}$ & $8,12 \mathrm{a}$ & $8,06 \mathrm{~b}$ \\
$(\%)$ & & & \\
Peso específico & $1,03 \mathrm{a}$ & $1,03 \mathrm{a}$ & $1,03 \mathrm{a}$ \\
$(\mathrm{g} / \mathrm{ml})$ & & & \\
\hline
\end{tabular}

$\mathrm{FVH}=$ forraje verde hidropónico, $\mathrm{AH}=$ alfalfa henificada, $\mathrm{VM}=$ vaina de mezquite.

Valores seguidos de diferente letra en el mismo renglón indican diferencia significativa según la prueba de Tukey $(\mathrm{P}<0,05)$.

forrajes, incrementando el contenido de ácidos grasos saturados y reduciendo el de los esenciales en la leche.

Referente al nivel proteico, las características energéticas y proteicas de la dieta que recibe el animal ejercen una mayor influencia, además de las condiciones genéticas del mismo, siendo tal vez la no degradabilidad de la proteína en el rumen el factor que modifica mayormente el contenido proteico de la leche. El mayor valor en la leche producida con la dieta AH-FVH 30\% (Cuadro 7) se debió al mayor contenido proteico de dicha dieta. El total proteico en la leche

Cuadro 7. Cantidad de sólidos producidos en leche de cabras suplementadas con tres diferentes dietas. Francisco I. Madero, Coahuila, México. 2011.

\begin{tabular}{llll}
\hline Parámetro* $^{*}$ & AH-FVH 15\% & AH-FVH 30\% & AH-VM \\
\hline Grasa $(\mathrm{g} / \mathrm{l})$ & $44,2 \mathrm{a}$ & $41,26 \mathrm{~b}$ & $38,42 \mathrm{~b}$ \\
Proteína $(\mathrm{g} / \mathrm{l})$ & $37,2 \mathrm{~b}$ & $38,7 \mathrm{a}$ & $37,6 \mathrm{~b}$ \\
\hline
\end{tabular}

$\mathrm{FVH}=$ forraje verde hidropónico, $\mathrm{AH}=$ alfalfa henificada, $\mathrm{VM}=$ vaina de mezquite.

Valores seguidos de diferente letra en el mismo renglón indican diferencia significativa según la prueba de Tukey $(\mathrm{P}<0,05)$.

* Gramos por cada 0,950 1 de leche, considerando que un litro de leche tiene una masa total de $1050 \mathrm{~g}$. 
depende en $60 \%$ de la proteína bacteriana producida en el rumen, y $40 \%$ de la aportada por los forrajes consumidos (Relling y Mattioli 2002).

\section{AGRADECIMIENTO}

Los autores agradecen al Consejo Estatal de Ciencia y Tecnología (COECYT) del estado de Coahuila, México, por el financiamiento para el presente proyecto.

\section{LITERATURA CITADA}

AOAC (Association of Official Analytical Chemistry). 2000. Official methods of analysis of the Association of Analytcal Chemists. 17 ed. Gaithersburg MD, USA. $2200 \mathrm{p}$.

Aregheore, EM; Ali, L; Ofori, K; Rere, T. 2006. Studies on grazing behavior of goats in the cook islands: The animal-plant complex in forage preference/palatability phenomena. Journal of Agriculture \& Biology 8:147-153

Boza, LJ; Sanz-Sampelayo, MR. 1997. Aspectos nutricionales de la leche de cabra. Edit. ACVAO. España. p. 109-139.

Chacón, VA. 2004. Acidez y peso específico de la leche de cabra de un grupo de capricultores de la Mesa Central Costarricense. Agronomía Mesoamericana 15:179182.

De La Rosa, CS. 2011. Manual de producción caprina. Capítulo 3. Edit. Formosa. Argentina. p. 4.

Elizondo J; Boschini, C. 2002. Producción de forraje con maíz criollo y maíz hibrido. Agronomía Mesoamericana 13:13-17.

Elizondo-Salazar, JA. 2008. Requerimientos nutricionales de cabras lecheras. I. Energía metabolizable. Agronomía Mesoamericana 19:115-122.

FAO (Organización de las Naciones Unidas para la Agricultura y la Alimentación). 2001. Manual técnico de producción de forraje verde hidropónico. TCP/ECU/066 (A) "Mejoramiento de la disponibilidad de alimentos en los Centros de Desarrollo Infantil del INNFA". Oficina Regional de La FAO Para América Latina y El Caribe. Santiago, Chile. 9 p.

García, HLA; Aguilar, A; Luévano, A; Cabral, A. 2005. La globalización productiva y comercial de la leche y sus derivados. Articulación de la ganadería intensiva lechera de la Comarca Lagunera. Editorial Plaza y Valdés. Colonia San Rafael, México. p. 11-13.

González, GJC; Ayala, BA; Gutiérrez, VE. 2006. Determinación de fenoles totales y taninos condensados en especies arbóreas con potencial forrajero de la región de Tierra Caliente, Michoacán, México. Livest Res Rural Dev 18:1-10.

Herrera, AAM; Depablos-Alviarez, LA; López, MR; Benezra-Sucre, MA; Ríos de Álvarez, La. 2007. Degradabilidad y digestibilidad de la materia seca del forraje hidropónico de maíz (Zea mays). Respuesta animal en términos de consumo y ganancia de peso. Revista Científica FCV-LUZ 17:372-379.

Lomeli, ZHM. 2000. Forraje verde hidropónico. El forraje del futuro...Hoy. Agrocultura 63:15-18.

López-Aguilar, R; Murillo-Amador, B; Rodríguez-Quezada, G. 2009. El forraje verde hidropónico $(\mathrm{FVH})$ : una alternativa de producción de alimentos para el ganado en zonas áridas. Interciencia 34:121-126.

Ludeña, F; Peralta, S; Arroyo, O; Fung, L; Gonzales, C. 2006. Caracterización fisicoquímica y microbiológica de la leche de cabra y su conservación mediante la activación del sistema lactoperoxidasa. Mosaico Cient. 3:17-26.

Mazorra, C; Fontes, D; Cubilla, N; Vega, A. 2009. Estrategias para modificar el consumo voluntario y la selección de alimentos de los pequeños rumiantes en pastoreo. Revista Cubana de Ciencia Agrícola 43:379-385.

NCR (National Research Council). 2007. Nutrient requirements of small ruminants: sheep, goats, cervids, and new world camelids. National Research Council. National Academy Press. Washington, DC, USA. 362 p.

Nunes, MA; Germano, AR; Batista, SI; Ramos, CFF; Vallecillo, A; Dos Santos, NM. 2007. Efecto de diferentes niveles dietéticos de heno de pasto elefante (Pennisetum purpureaum, Schum Var. Cameroon) para la recría de caprinos. Arch. Latinoam. Prod. Anim. 3:71-78.

Pinto, CM; Carrasco, RE; Fraser, LB; Letelier, HA; Dorner, PW. 1998. Composición química de la leche cruda y sus variaciones a nivel de silos en plantas lecheras de la VIII, IX, y X Regiones de Chile. Parte I. Macrocomponentes. Agrosur (en línea). Consultado octubre 2012. Disponible en http://mingaonline.uach.cl/scielo. php?script=sci_arttext $\&$ pid $=$ SO304_8802199800020 0010\&Ing=es\&nrm=iso $>$ ISSN0304-8802.

Relling, AE; Mattioli, GA. 2002. Fisiología digestiva y metabolismo de los rumiantes. Fac. Cs. Veterinarias. UNLP. Universidad Nacional La Plata. ED. EDULP. Argentina. p. 34-38. 
Revidatti, MA; De La Rosa, SA; Capello-Villada, JS; Orga, A. 2012. Indicadores productivos de hembras caprinas en el oeste de Formosa Argentina. AICA 2:75-81.

Rivera, A; Moronta, M; González, EM; González .; Perdomo D; García, DE; Hernández, G. 2010. Producción de forraje hidropónico de maíz (Zea mays L.) en condiciones de iluminación deficiente. Zootecnia Tropical 28:33-41.

Rodríguez, SAC. 2003. Como producir con facilidad, rapidez y óptimos resultados forraje verde hidropónico. Ed. Diana. México. p. 69-70.

Romero-Valdez, ME; Córdova-Duarte, G; Hernández-Gallardo, EO. 2009. Producción de forraje verde hidropónico y su aceptación en ganado lechero. Acta Universitaria 19:11-19.

Salas-Pérez, L; Preciado-Rangel, P; Esparza-Rivera, JR; Álvarez-Reyna, V; Palomo-Gil, A; Rodríguez-Dimas, N; Márquez-Hernández, C. 2010 Rendimiento y calidad de forraje hidropónico producido bajo fertilización orgánica. Terra Latinoamericana 28:355-360.

Salas-Pérez, L; Esparza-Rivera, JR; Preciado-Rangel, P; Álvarez-Reyna, V; Meza-Velázquez, JA; VelázquezMartínez, JR; Murillo-Ortiz, M. 2012. Rendimiento, calidad nutricional, contenido fenólico y capacidad antioxidante de forraje verde hidropónico de maíz (Zea mays) producido en invernadero bajo fertilización orgánica. Interciencia 37:215-220.

Sánchez, C; García, M; Alvarez, M. 2003. Efecto de la suplementación alimenticia sobre el comportamiento productivo de cabras al postparto en la microrregión Río Tocuyo, Estado Lara. Zootecnia Trop. 21:43-55.
SAS (Statistical Analysis System). 1999. SAS/STAT User's guide: Statistics. Version 9.0. Cary, NC, USA.

SIAP (Servicio de Información Agroalimentaria y Pesquera). 2010. Producción ganadera en la Comarca Lagunera. (en línea). Consultado ene. 2012. Disponible en http:// www.siap.gob.mx/index.php?option=com_content $\& v$ iew $=$ article $\&$ id $=3 \&$ Itemid $=29$

SIAP (Servicio de Información Agroalimentaria y Pesquera). 2011. Producción de forrajes en la Comarca Lagunera (en línea). Consultado enero 2012. Disponible en http://www.siap.gob.mx/index.php?searc hword=forrajes \&ordering $=\&$ searchphrase $=$ all $\&$ Ite mid=518\&option=com_search

Steel, R; Torrie, J. 1980. Principles and procedures of statistics: Abiometral approach. USA. 2 ed. Ed. McGrawHill. USA. 633 p.

Valdivia, BE. 1996. Producción de forraje verde hidropónico $(\mathrm{FVH})$. Curso Taller Internacional de Hidroponía. Lima, Perú. p. 201-206.

Van Soest, PJ; Mertens, DR; Deinum, B. 1991. Preharvest factors influencing quality of conserved forage. $\mathrm{J}$. Anim. Sci. 47:712-720.

Vargas, RCF. 2008. Comparación productiva de forraje verde hidropónico de maíz, arroz y sorgo negro forrajero. Agronomía Mesoamericana 19:233-240.

Villanueva, DJ; Cerano, PJ; Constante, GV; Stahl, DW; Estrada, AJ; Tostado, PMM. 2011. Variabilidad hidroclimática histórica del norte de México inferida con anillos de crecimiento de Douglas-Fir. Revista Mexicana de Ciencias Agrícolas 2:221-234. 